\title{
CONTROLE DA INFESTAÇÃO NATURAL DE Ceratitis capitata (WIED., 1824) (DIPTERA, TEPHRITIDAE) EM PÊSSEGOS (Prunus persica) ATRAVÉS DAS RADIAÇÕES GAMA
}

\author{
ARTHUR, V.; CACERES, C.; WIENDL, F.M. \\ Centro de Energia Nuclear na Agricultura/USP, C.P. 96, CEP: 13400-970-Piracicaba,SP \\ WIENDL, J.A. \\ Informaut, Informática Mattiolli, Av. Carlos Botelho, 638, CEP: 13416-145 - Piracicaba, SP
}

\begin{abstract}
RESUMO: Determinou-se a dose desinfestante de radiações gama para pêssegos, Prunus persica, infestados com larvas da mosca do Mediterrâneo, Ceratitis capitata. Utilizaram-se frutas de procedéncia conhecida no campo fazendo-se uma amostragem prévia, constatando-se que cada fruta continha em média nove larvas do último ínstar da mosca praga. As frutas foram irradiadas em uma fonte de Cobalto-60 com as seguintes doses de radiação gama: 0 (test.), 25, 50, 100, 200, 400, 600, 800, 1000 e $1200 \mathrm{~Gy}$, sob uma taxa de $58 \mathrm{~Gy}$ por minuto. Após a irradiação as frutas foram colocadas em camaras climatizadas com a temperatura variando entre 23 e $27^{\circ} \mathrm{C}$ e a umidade relativa variando entre 65 e $75 \%$. Aguardou-se que as larvas deixassem as frutas e se transformassem em pupas e adultos. A dose letal para larvas, pelos resultados obtidos no experimento, concluiu-se ser de $600 \mathrm{~Gy}$. A dose letal para pupas provenientes de larvas irradiadas dentro das frutas foi de $50 \mathrm{~Gy}$, impedindo totalmente a emergência de adultos.
\end{abstract}

Descritores: irradiação de alimentos, mosca do Mediterrâneo, quarentena, Ceratitis capitata, Prunus persica

\section{CONTROL OF NATURALLY INFESTED PEACHES (Prunus persica) BY MEDITERRANEAN FRUIT FLY (Ceratitis capitata) THROUGH THE USE OF GAMMA RADIATION}

\begin{abstract}
Determination of the dose of gamma radiation to disinfest peaches, Prunus persica infested with larvae of Ceratitis capitata (Wied., 1824) was made. Fruits were collected in the field, each one holding about nine larvae of the last instar of the fruit-fly. The fruits were irradiated with Cobalt- 60 gamma radiation source at the following doses: 0 (control), 25, 50, 100, 200, 400, 600, 800, 1000 and $1200 \mathrm{~Gy}$; at a dose rate of $58 \mathrm{~Gy}$ per minute. After irradiation the fruits were kept in a climatic chamber with the temperature adjusted between 23 and $27^{\circ} \mathrm{C}$, and relative humidity between 65 and 75 percent, until the larvae left the fruits and were transformed into pupae and adults. It was concluded that the lethal dose of gamma radiation for larvae at the last instar, in naturally infested peaches, was $600 \mathrm{~Gy}$ and the dose of $50 \mathrm{~Gy}$ inhibited completely the emergency of adults.
\end{abstract}

Key Words: food irradiation, peach disinfestation, Mediterranean fruit-fly, Ceratitis capitata, Prunus persica.

\section{INTRODUÇÃO}

A produção nacional das frutas tradicionais apresentou um grande aumento nos últimos anos, e vem desempenhando papel cada vez mais importante no campo da comercialização no exterior. Assim, em face da crescente demanda dos países importadores e da potencialidade do Brasil para atendê-la, vem sendo requerido o estabelecimento de condições favoráveis ao desenvolvimento do setor de exportação, assim como do consumo interno.

Uma das espécies pragas mais frequentes e de maior importância econômica para os fruticultores é a mosca do Mediterrâneo Ceratitis capitata (Wied., 1824). As larvas desta mosca alimentam-se da polpa das frutas, facilitando - ataque de podridóes e a queda prematura dos frutos infestados, que se perdem irremediavelmente.

Além dos danos diretos, essa praga traz problemas para a exportação dos frutos "in natura", uma vez que os países consumidores, livres dela, aplicam rigorosas leis de quarentena, exigindo um tratamento eficaz de desinfestação.

Nos Estados Unidos a Agência de Proteção Ambiental proibiu em 1984 o uso do Dibrometo de Etileno (EDB), fumigante normal- 
mente usado na desinfestação de alimentos MOY et al., (1986). Assim, a fim de satisfazer o mercado consumidor exigem-se tratamentos alternativos.

Em 1982, BURDITT \& SEO concluiram que o uso das radiações gama é indicado como tratamento de quarentena para produtos infestados com ovos e larvas de moscas-das-frutas.

A irradiação possue as seguintes vantagens em relação ao tratamento com produtos químicos, tratamentos térmicos ou combinação de ambos: segundo MOY et al. (1983) é um processo contínuo e totalmente eficiente, assegurando completa desinfestação dos produtos; não deixa resíduos nos frutos e tende a retardar o amadurecimento das frutas climatéricas, aumentando o tempo de armazenamento sem deterioração.

$O$ objetivo deste trabalho foi determinar a dose de desinfestação de pêssegos atacados por larvas de $C$. capitata através da radiação gama do Cobalto-60, para satisfazer as necessidades quarentenárias de países importadores.

\section{Revisão Bibliográfica}

A possibilidade do uso de radiação gama como tratamento de desinfestação de frutas atacadas por moscas-das-frutas, foi proposta por BALOCK et al. (1956) e desde então muitas pesquisas vem sendo realizadas visando implementar a aplicabilidade deste método.

No Brasil, GALLO (1960) foi o primeiro a utilizar a radiação gama para irradiar pupas da mosca-do-Mediterrâneo Ceratitis capitata (Wied.) com o fim de esterilizar os insetos adultos, objetivando a aplicação da Técnica do Inseto Estéril nas plantações cítricas. Posteriormente, BALOCK et al. (1963), irradiaram ovos, larvas e pupas de C. capitata e concluiram que a dose de $20 \mathrm{krad}$ era eficiente no tratamento de quarentena para desinfestação de frutas frescas. Por outro lado, FESUES et. al. (1981) concluiram que doses entre 40 e $60 \mathrm{krad}$ de radiação gama eram capazes de induzir a desinfestação de laranjas atacadas por $C$. capitata, sem causar injúrias aos frutos. Entretanto, SHEHATA (1983) concluiu que doses entre $84 \mathrm{e}$ $94 \mathrm{krad}$ de radiação gama foram suficientes para desinfestar $C$. capitata de frutos atacados.

DOMARCO et al. (1989) irradiaram mangas e mamões com doses crescentes de radiação gama com o objetivo de desinfestar as frutas atacadas por larvas de $C$. capitata. Pelos resultados obtidos concluiram que a dose de 900
Gy foi suficiente para desinfestar essas frutas.

POTENZA et al. (1989) mostraram que a dose de 40 Gy foi suficiente para inibir a emergência de adultos de $C$. capitata no interior de mangas, quando irradiados na fase larval com idade de até 7 dias. Posteriormente RAGA et al. (1991) determinaram que a dose de $\mathbf{4 0}$ Gy foi suficiente para inibir a emergência de adultos de $C$. capitata em laranjas Valência quando irradiadas em sua fase larval na idade de 4 dias.

Recentemente, COSTA et al. (1993a) determinaram que a dose de 50 Gy foi suficiente para causar esterilização em todas as fases imaturas de C. capitata em laranjas e tangerinas. Os mesmos autores, COSTA et al. (1993b), determinaram que a dose de $400 \mathrm{~Gy}$ foi suficiente para impedir a sobrevivência de qualquer fase imatura de $C$. capitata em laranjas e tangerinas.

ARTHUR et al. (1993) irradiaram com doses crescentes de radiação gama pêssegos artificialmente infestados com larvas da mosca do Mediterrâneo. Pelos resultados obtidos concluiram que a dose letal para larvas foi de 500 Gy e aquela que impediu a emergência de adultos foi de 50 Gy de radiações gama.

\section{MATERIAIS E MÉTODOS}

O experimento foi realizado nos laboratórios da Seção de Entomologia do Centro de Energia Nuclear na Agricultura-CENA da Universidade de São Paulo-USP em Piracicaba, São Paulo.

Para a realização do experimento utilizaram-se pêssegos de procedência conhecida sendo que se fez uma amostragem prévia da infestação latente, averiguando-se que cada pêssego continha uma média de nove larvas de C.capitata do último instar. Esses pêssegos foram irradiados em uma fonte de Cobalto- 60 , tipo Gammabeam 650 e sob uma taxa de dose de aproximadamente 58 Gy/hora. As doses empregadas foram: 0 (test.), $25,50,100,200,400,600,800,1000$ e $1200 \mathrm{~Gy}$. Cada tratamento constou de 3 repetições com 5 frutas por repetição ou seja 45 larvas por dose.

Após a irradiação esses pêssegos foram colocados em sacos plásticos de aproximadamente meio litro de capacidade, nos quais foram feitos pequenos orificios para que houvesse trocas gasosas. No interior dos sacos plásticos foi colocado um volume de aproximadamente $80 \mathrm{ml} \mathrm{de}$ bagacilho de cana, onde as larvas, após deixarem os frutos, pudessem se transformar em pupas. 
TABELA 1 - Número médio de pupas e emergência de adultos de Ceratitis capitata provenientes de pêssegos infestados e irradiados com radiações gama do Cobalto- 60 .

\begin{tabular}{cccccc}
\hline \hline & & & & \multicolumn{2}{c}{ Emergência de adultos } \\
\cline { 5 - 6 } Tratamento & & $\mathrm{n}^{0}$ total pupas & $\mathrm{n}^{\circ}$ larvas mortas & machos & fêmeas \\
\hline 0 & $\mathrm{~m}$ & $33.0 \mathrm{a}$ & $3.7 \mathrm{a}$ & $4.7 \mathrm{a}$ & $6.3 \mathrm{a}$ \\
25 & $\mathrm{~m}$ & $30.7 \mathrm{a}$ & $4.7 \mathrm{a}$ & $0.3 \mathrm{c}$ & $0.3 \mathrm{c}$ \\
50 & $\mathrm{~m}$ & $28.3 \mathrm{a}$ & $5.0 \mathrm{a}$ & $0.0 \mathrm{c}$ & $0.0 \mathrm{c}$ \\
100 & $\mathrm{~m}$ & $5.3 \mathrm{~b}$ & $12.7 \mathrm{~b}$ & $0.0 \mathrm{c}$ & $0.0 \mathrm{c}$ \\
200 & $\mathrm{~m}$ & $2.7 \mathrm{~b}$ & $12.0 \mathrm{~b}$ & $0.0 \mathrm{c}$ & $0.0 \mathrm{c}$ \\
400 & $\mathrm{~m}$ & $2.3 \mathrm{~b}$ & $14.7 \mathrm{~b}$ & $0.0 \mathrm{c}$ & $0.0 \mathrm{c}$ \\
600 & $\mathrm{~m}$ & $0.0 \mathrm{~b}$ & $15.3 \mathrm{~b}$ & $0.0 \mathrm{c}$ & $0.0 \mathrm{c}$ \\
800 & $\mathrm{~m}$ & $0.0 \mathrm{~b}$ & $18.3 \mathrm{~b}$ & $0.0 \mathrm{c}$ & $0.0 \mathrm{c}$ \\
1000 & $\mathrm{~m}$ & $0.0 \mathrm{~b}$ & $16.7 \mathrm{~b}$ & $0.0 \mathrm{c}$ & $0.0 \mathrm{c}$ \\
1200 & $\mathrm{~m}$ & $0.0 \mathrm{~b}$ & $21.7 \mathrm{c}$ & $0.0 \mathrm{c}$ & $0.0 \mathrm{c}$ \\
\hline \hline
\end{tabular}

Letras iguais significam semelhança estatística a nível de 95\% (Tukey)

Posteriormente separou-se as pupas do bagacilho, colocando-as em tubos de vidro com tampas de algodão, e aguardou-se a emergência dos adultos. O experimento foi realizado em uma câmara climatizada com temperatura regulada entre 23 e $27^{\circ} \mathrm{C}$ e com umidade relativa entre 65 e $75 \%$.

\section{RESULTADOS E DISCUSSÃo}

Constam na TABELA 1 as médias dos dados obtidos nas três repetições, onde se relaciona a média do número de larvas que deixaram os frutos e se transformaram em pupas e em adultos. Pela TABELA 1 observa-se que nas doses acima de $50 \mathrm{~Gy}$ a radiação gama influenciou significativamente o desenvolvimento das larvas, diminuindo sua transformação em pupas. Já a dose de $600 \mathrm{~Gy}$, foi totalmente letal para larvas, sendo que a dose de $50 \mathrm{~Gy}$ inibiu completamente a emergência dos adultos. Estes resultados estão de acordo com os obtidos por POTENZA et al. (1989), RAGA et al. (1991), COSTA et al. (1993b) e ARTHUR et al. (1993), mas bem inferiores aos obtidos por DOMARCO et al. (1989).

\section{CONCLUSÕES}

Concluiu-se que para fins de desinfestação quarentenária através da radiação gama do Cobalto-60, frutas de pêssego infestadas por C. capitata e irradiadas com a dose de $50 \mathrm{~Gy}$ tornaram-se perfeitamente seguras para exportação, pois a radiação inibe completamente a emergência de adultos desta mosca.

\section{REFERÊNCIAS BIBLIOGRÁFICAS}

ARTHUR, V.; WIENDL F.M.; WIENDL, J.A. Controle de Ceratitis capitata (Wied., 1824) (Diptera, Tephritidae) em pêssegos (Prunus persica) infestados artificialmente, através das radiações gama do Cobalto-60. Revista de Agricultura, Piracicaba, 1993. (No prelo).

BALOCK, J.W.; BURDITT JR., A.K.; CHRISTENSON, L.D. Effects of gamma radiation on various stages of three fruit-fly species. Journal of Economic Entomology, New Hampshire, v.56, n.1, p.42-46, 1963. 
BALOCK, J.W.; CHRISTENSON, L.D.; BURR, G.O. Effects of gamma rays from Cobalt-60 on immature stages of the oriental fruit-fly Dacus dorsalis and possible application to commodity treatment problems. In: ANNUAL MEETING OF THE HAWAII ACADEMY SCIENCE, 22,, 1956, Honolulu. Proceedings Annual Academy Science, v.31, 18p. 1956.

BURDITT JR., A.K. \& SEO, S.T. Dose requirements for quarantine treatment of fruit flies with gamma irradiaton. In: SEMINAR ON FOOD IRRADIATION FOR DEVELOPING COUNTRIES IN ASIA AND THE PACIFIC, 1982. Tokyo. Report... Vienna: AIEA, 1982. p.57-87.

COSTA, N.; ARTHUR, V; COSTA, M.L.Z.; ITEPAN, N.M.; WIENDL, F.W.; WIENDL, F.M. Determinação da dose esterilizante de radiação gama (Co-60) para Ceratitis capitata (Wied., 1824) em laranjas e tangerinas. In: CONGRESSO BRASILEIRO DE ENTOMOLOGIA, 14., 1993. Piracicaba. Resumos... Piracicaba: SEB, 1993b. v.1, p.582.

COSTA, N.; ARTHUR, V.; COSTA, M.L.Z.; ITEPAN, N.M.; WIENDL, F.W.; WIENDL, F.M. Irradiação de laranjas e tangerinas atacadas pela mosca-do-Mediterrâneo Ceratitis capitata (Wied., 1824) através de fonte de Cobalto-60, para fins de desinfestaçāo. In: CONGRESSO BRASILEIRO DE ENTOMOLOGIA, 14., 1993. Piracicaba. Resumos... Piracicaba: SEB, 1993a. v.1, p.585.

DOMARCO, R.E.; WALDER, J.M.; ARTHUR, V.; WIENDL, F.M. Desinfestação de mangas (Mangifera indica L.) e mamões (Carica papaya L.) contra Ceratitis capitata (Wied., 1824), através da radiação gama. In: CONGRESSO BRASILEIRO DE ENTOMOLOGIA, 12., 1989. Belo Horizonte. Resumos... Belo Horizonte: SEB, 1989. v.2, p.501.

FESUES, I.; KADAS, L.; KALMAN, B. Protection of oranges by gamma radiation against Ceratitis capitata (Wied.). Acta Alimentaria, Budapest, v.10, n.4, p.293-299, 1981.
GALLO, D. Radioisótopos no controle de pragas. $O$ Solo, Piracicaba, v.1, p.30-31, 1960.

MOY, J.H.; KANESHIRO, K.Y.; OHTA, A.T.; NAGAI, N.Y. Radiation disinfestation of California stone fruits infested by Medfly: effectiveness and fruit quality. Journal of Food Science, Chicago, v.48, n.3, p.928-934, 1983.

MOY, J.H.; REYES, L.J.; RAMIREZ, G.T.; BUSTOS, R.E. The economics of radiation disinfestation of Mexican mangoes and citrus as quarantine treatment. In: ANNUAL MEETING OF THE INSTITUTE OF FOOD TECHNOLOGISTS, 1986. Dallas. Proceedings... Dallas: Institute of Food Tecnologists, 1986. p.231-42.

POTENZA, M.R.; YASUO-KA, S.T.; GIORDANO, R.B.P.; RAGA, A. Irradiação de frutos de laranja infestados com larvas da mosca das frutas Ceratitis capitata (Wied., 1824). In: CONGRESSO BRASILEIRO DE ENTOMOLOGIA, 12., 1989. Belo Horizonte. Resumos... Belo Horizonte: SEB, 1989. v.2, p.509.

RAGA, A.; SATO, M.E; GIORDANO, R.B.P.; POTENZA, M.R.; SZULAK, C.; SUPLICY FILHO, $N$. Uso da radiação gama na desinfestação de mangas destinadas a exportação em relação a larvas de Ceratitis capitata, Anastrepha fraterculus e Anastrepha obliqua. In: CONGRESSO BRASILEIRO DE ENTOMOLOGIA, 13,, 1991. Rio de Janeiro. Resumos... Rio de Janeiro: SEB, 1991. v.2, p.632.

SHEHATA, N.P. Effect of gamma radiation on the immature stages of the Mediterranean fruit-fly, Ceratitis capitata (Wied.). Isotope and Radiation Research, Cairo, v.15. n.12, p.121-129, 1983.

Enviado para publicação em 29.07 .93

Aceito para publicação em 29.08.93 